\title{
Fast and Efficient Discrimination of Traveling Salesperson Problem Stimulus Difficulty
}

\section{Matthew J. Dry' and Elizabeth L. Fontaine ${ }^{1}$}

\author{
'School of Psychology, University of Adelaide, Australia
}

\section{Correspondence:}

Matthew J. Dry. Phone: +618 8313 3856;

E-mail: matthew.dry@adelaide.edu.au

Keywords:

problem solving, optimization, perceptual processing, geometric minimality, prägnanz
The Traveling Salesperson Problem (TSP) is a computationally difficult combinatorial optimization problem. In spite of its relative difficulty, human solvers are able to generate close-to-optimal solutions in a close-to-linear time frame, and it has been suggested that this is due to the visual system's inherent sensitivity to certain geometric properties of TSP stimuli. In the current study we employed a novel experimental paradigm in which we presented participants with sets of four TSP stimuli that varied in terms of their relative solution difficulty and asked them to indicate which of the four stimuli they would prefer to solve. The results indicated that the participants' choice frequencies followed the same ordering as the stimuli's empirical solution difficulty; that is, easy-to-solve stimuli were chosen with a higher frequency than hard-to-solve stimuli. It is suggested that these results provide further evidence of the speed and efficiency of human processing of TSPs, and provide further evidence implicating the role of rapid visuo-perceptual organization in generating TSP solutions. An analysis of the geometric properties of the stimuli uncovered a number of factors that may have influenced the choice preferences of participants in the current experiment, and the performance quality of participants in previous experiments.
The Traveling Salesperson Problem (TSP) is a difficult combinatorial optimization problem that has been studied across many different disciplines including engineering, computer science, mathematics, and more recently, psychology. The TSP can be formalized in numerous ways (Garfinkel, 1985), but in the context of psychological research it generally consists of a set of nodes or 'cities' distributed within a two-dimensional Euclidean plane (e.g., Figure 1, left). The solver is required to find the shortest possible pathway that passes through each node once and once only, and in the case of the more common 'closed' version of the task, returns to the start-node. The optimal solution to the stimulus in Figure 1 is shown in the right-hand panel. The majority of psychological research has focused upon the two-dimensional Euclidean version of the problem, however Walwyn and Navarro (2010), and Haxhimusa et al. (2011) provide recent examples of psychological studies employing non-Euclidean and higher dimensional TSP variants. In the following, unless specifically indicated otherwise, we will use the term TSP to refer to the two-dimensional Euclidean closed-form of the problem.

One of the reasons that the TSP is of interest is that it is a computationally difficult problem. For any given TSP of size $n$ there are $(n-1) ! / 2$ potential solutions-thus the number of potential solutions increases rapidly as problem size increases. For example, while a 5-node problem has only 12 potential solutions, a 10 -node problem has 181,440 , and a 15 -node problem has $4.35 \times 10^{10}$ potential solutions. Importantly, to date there is no known computer algorithm that is able to find the optimal solution to TSPs in a practical polynomial timeframe.

In spite of the apparent computational complexity of the TSP empirical evidence has shown that human solvers are remarkably adept at solving this problem. For example, human solvers are able to generate close-to-optimal solutions to the TSP in a linear, or close-to-linear time frame (e.g., Dry, Lee, Vickers, \& Hughes, 2006; Graham, Joshi, \& Pizlo, 2000). Furthermore, while the degree to which human solutions exceed the optimal solution also increases as a function of $n$, the pattern of change is asymptotic and shows little increase in deviation from optimality as problem size increases from 80 to 120 nodes (Dry et al., 2006). Additionally, human performance on the task has been shown to compare well to various different computational approaches that (unlike

Figure 1.

A randomly distributed stimulus (left) and the associated optimal TSP solution (right).

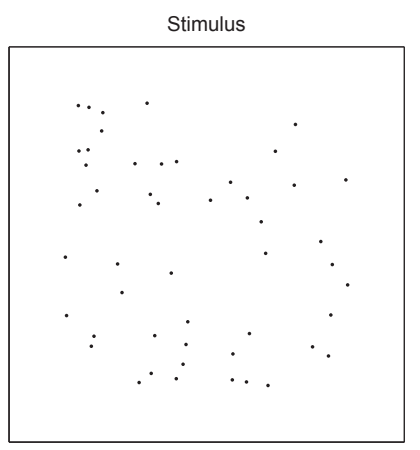

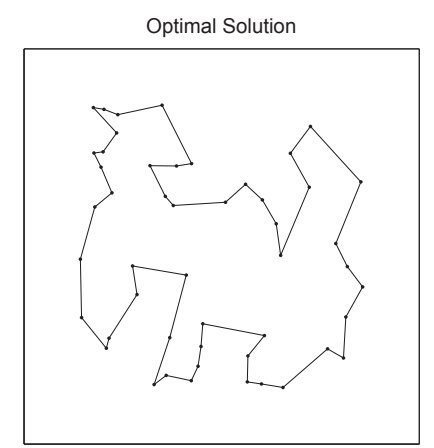




\section{Figure 2.}

Example TSP stimuli belonging to classes that have varying relative solution difficulty (see text for details).
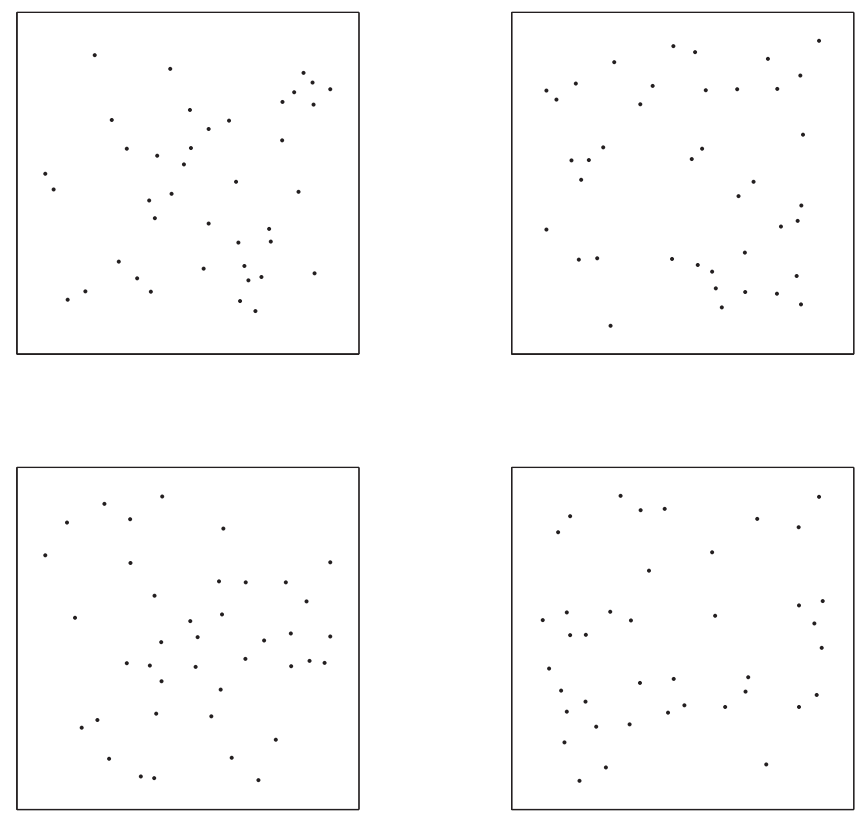

Figure 3.

A randomly distributed TSP stimulus and all of the potential edges that could be used in generating a solution.

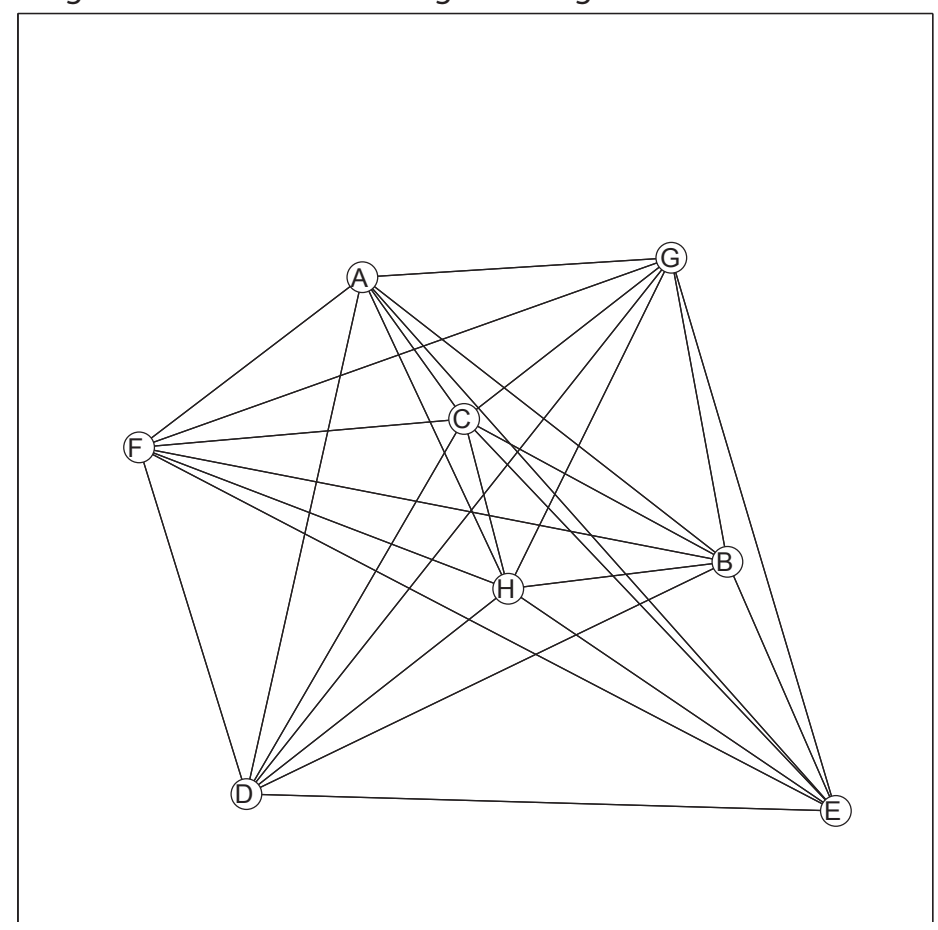

human solvers) have access to the actual inter-node distances and related geometric properties of the stimuli (e.g., Best, 2005; Graham, et al., 2000; Haxhimusa, et al., 2011; Haxhimusa, Kropatsch, Pizlo, \& Ion, 2009; Hill, 1982; MacGregor \& Ormerod, 1996; MacGregor, Ormerod, \& Chronicle, 1999, 2000; Pizlo et al., 2006; Tak, Plaiser, \& van Rooij, 2008; Vickers, Butavicius, Lee, \& Medvedev, 2001).

\section{RATIONALE AND OUTLINE}

In this paper we present a novel experimental paradigm that provides additional evidence of the speed and efficiency of human performance on the TSP. In our experiments, instead of asking participants to just solve TSPs, we presented them with a range of stimuli (such as can be seen in Figure 2) that had previously been shown to influence the performance of human solvers, and asked them to choose the stimulus that they believed would be easiest to solve.

We were interested to see if there were any meaningful patterns in the choice preferences of the participants-specifically, whether they matched the pattern of previously demonstrated solution difficulty. We reasoned that if the participants demonstrated a preference for choosing the 'easier' stimuli then this would provide further evidence of the speed and efficiency of human performance on the TSP. In other words, being able to make meaningful discriminations would suggest the possibility that the participants were able to generate multiple TSP solutions (or partial solutions) to the stimuli, store these in working memory, and compare them to each other in terms of either perceived optimality, or ease of solution generation (or both). Such a result would suggest that the processes underlying human performance on the TSP are even more computationally powerful than previously assumed.

In the following sections we present two experiments providing evidence that suggests human observers are able to meaningfully discriminate between different TSP stimuli in regards to their solution difficulty. Following this we provide an analysis of the stimuli in an attempt to further understand the properties that influenced the choice preferences of participants in the current experiment, and the performance quality of participants in previous experiments. Finally, we address the wider implications of these findings in a general discussion.

\section{EXPERIMENT ONE}

As indicated, the aim of the current experiment was to determine if participants were able to discriminate between different TSP stimuli in terms of their relative solution difficulty. The stimuli employed in the current study were taken from Vickers, Lee, Dry, and Hughes (2003). In this study participants were asked to solve TSP stimuli that had been manipulated in terms of two geometric properties: number of nodes on the convex hull, and number of intersections. These two properties are demonstrated in Figure 3, which presents an example of a TSP stimulus superposed with all of the edges that can be used to connect the stimulus nodes. The convex hull is the shortest enclosing polygon of a given point set in the plane, and stimulus nodes either fall within this polygon, or lie on the hull. In Figure 3 the nodes A-G-E-D-F comprise 


\section{Figure 4.}

Deviation from optimal and response uncertainty data for the $\mathrm{L}_{\text {int }}$ \& $\mathrm{L}_{\text {node }} \mathrm{H}_{\text {int }}$ \& $\mathrm{L}_{\text {node }} \mathrm{H}_{\text {int }}$ \& $\mathrm{H}_{\text {node' }}$ and $\mathrm{L}_{\text {int }}$ \& $\mathrm{H}_{\text {node }}$ stimulus conditions from Vickers et al. (2003).

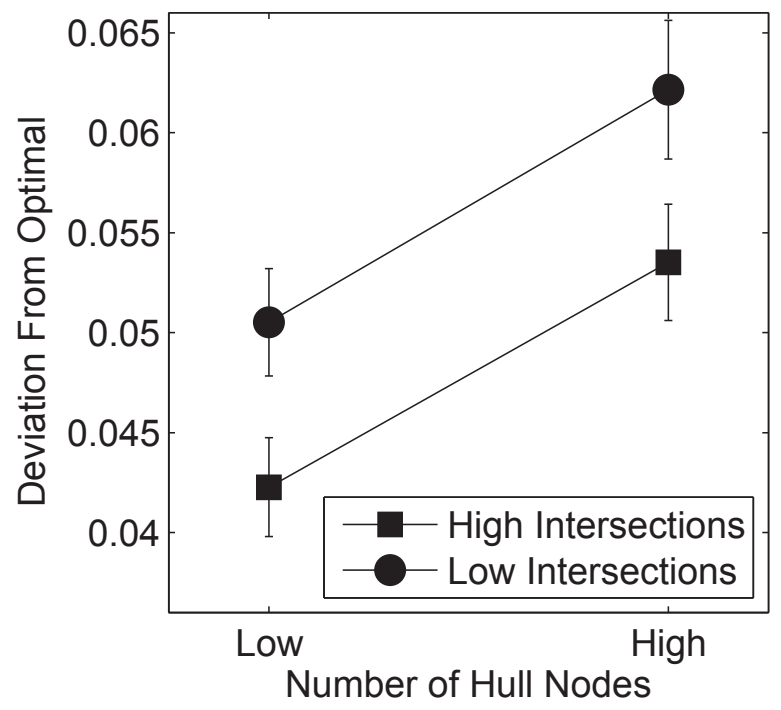

the set of nodes that lie on the convex hull. The number of intersections is simply a count of the number of edges that intersect with another edge-in this case 21 edges intersect with another edge.

These two properties are of theoretical interest as they are believed to provide insight into the processes underlying human performance on TSPs. For example, it has been suggested that the convex hull provides an initial outline for a solution, and the interior nodes are then incorporated into this sub-tour to form the final solution (e.g., MacGregor \& Ormerod, 1996; MacGregor et al., 2000). If this were the case then it could be expected that there would be a relationship between stimulus difficulty and the number of convex hull nodes (proportional to the total number of stimulus nodes). In other words, we could expect that difficulty would increase as the ratio of convex hull to interior nodes decreases.

In regards to the second of these properties it has been reasoned that the number of intersecting edges within a stimulus places a constraint upon the number of viable potential solutions that need to be considered (e.g., Vickers et al., 2003), because solutions with intersections will always be sub-optimal (e.g., van Rooij, Stege, \& Schactman, 2003). In other words, a stimulus with a high number of intersecting edges will have fewer non-intersecting potential solutions, and should therefore be easier to solve than a stimulus with a low number of intersecting edges. It should be noted that it is possible to make an argument for the opposite relationship-if participants are actively avoiding intersections (as suggested by the van Rooij et al., 2003, 'crossing avoidance' strategy) then problem difficulty should increase as the number of potential intersections increases. In other words, the difficulty of avoiding intersections would increase as the number of intersections to be avoided increased. ${ }^{1}$

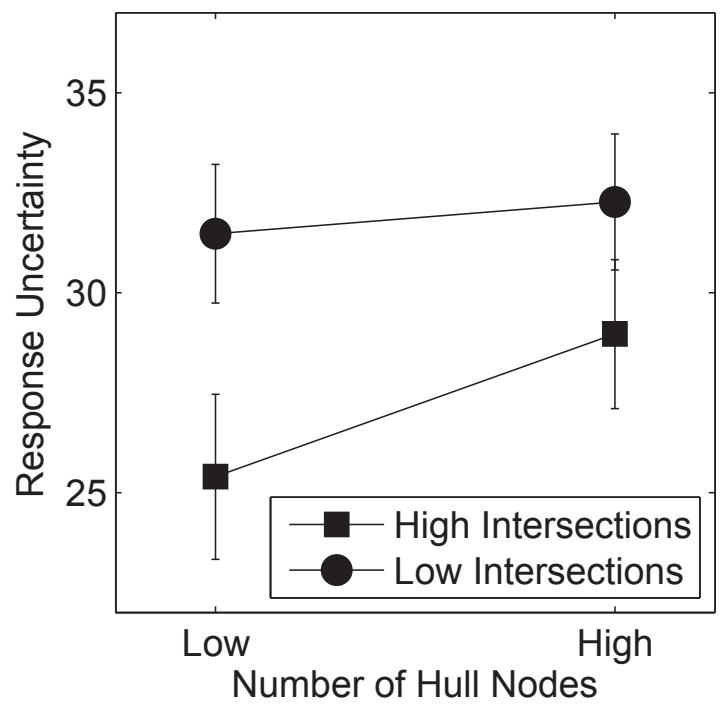

The Vickers et al. (2003) experiment employed stimuli that were classified as having a high, medium or low number of convex hull nodes and a high, medium or low number of intersections. In the current experiment we employ only four of these stimulus classes: low number of intersections and low number of hull nodes (which we will denote as $\mathrm{L}_{\text {int }}$ $\left.\& L_{\text {node }}\right)$, high number of intersections and low number of hull nodes $\left(\mathrm{H}_{\mathrm{int}} \& \mathrm{~L}_{\text {node }}\right)$, high number of intersections and high number of hull nodes $\left(\mathrm{H}_{\mathrm{int}} \& \mathrm{H}_{\text {node }}\right)$, and low number of intersections and high number of hull nodes $\left(\mathrm{L}_{\text {int }} \& \mathrm{H}_{\text {node }}\right)$. Examples of these stimulus classes are shown in Figure 2. Moving clockwise from the top left the stimuli are $\mathrm{L}_{\text {int }} \& \mathrm{~L}_{\text {node' }}$, $\mathrm{H}_{\text {int }} \& \mathrm{~L}_{\text {node }}, \mathrm{H}_{\text {int }} \& \mathrm{H}_{\text {node }}$, and $\mathrm{L}_{\text {int }} \& \mathrm{H}_{\text {node }}$, respectively.

The results of Vickers et al. (2003) indicated that both of these factors had an effect upon solution quality, measured as either the degree to which the length of empirical solutions exceeded the length of benchmark optimal solutions (deviation from optimality) or the degree of agreement across the participant's solutions (response uncertainty). Figure 4 shows the results of Vickers et al. (2003) re-plotted showing only the stimulus conditions employed in the current study. To summarize: participants generated better solutions to stimuli with a high number of intersections compared to stimuli with a low number of intersections, and better solutions to stimuli with a low number of nodes on the convex hull compared to stimuli with a high number of nodes on the convex hull. ${ }^{2}$

In regards to the current experiment, we assumed that if participants were aware of the relative difficulty of the different stimuli then we could expect to see a pattern of results similar to that in Figure 4: participants should indicate that stimuli with a high number of intersections would be easier to solve than stimuli with a low number of intersections, and stimuli 
with a low number of nodes on the convex hull would be easier to solve than stimuli with a high number of nodes on the hull.

\section{METHOD}

\section{Participants}

The participants $(\mathrm{N}=37)$ were 21 males and 16 females, with a mean age of 29 years. They either performed the experiment for partial course credit or were paid \$15 AUS. All participants provided informed consent, and the study was approved by the School of Psychology's human ethics subcommittee.

\section{Stimuli}

As indicated in Vickers et al. (2003), the stimuli were selected as follows: First, they generated a pool of 20,000 randomly distributed 40 node stimuli. Then, for each stimulus, they measured the number of intersections and the number of nodes on the convex hull. The mean number of intersections was 63,542-based upon this they set the medium number of intersections to be equal to the mean, and the high and low number of intersections to 1.5 standard deviations above and below this mean $(66,341$ and 60,743 for high and low, respectively). The median number of nodes on the hull was 9.5based on this the low number of nodes was set to 7 and high to 12 (with medium alternating equally between 9 and 10). Given these values (and a tolerance of $+/-93$ for the number of intersections) Vickers et al. (2003) randomly chose 8 stimuli that matched these criteria in a 3 (low, medium, and high intersections) $\times 3$ (low, medium, and high convex hull nodes) factorial design, resulting in a total of 72 stimuli.

As indicated earlier, the current study only employed a sub-set of these stimulus classes in the main experimental task (e.g., the $8 \mathrm{~L}_{\text {int }} \& \mathrm{~L}_{\text {node }}, 8 \mathrm{H}_{\text {int }} \& \mathrm{~L}_{\text {node }}, 8 \mathrm{H}_{\text {int }} \& \mathrm{H}_{\text {node }}$, and 8 $\mathrm{L}_{\text {int }} \& \mathrm{H}_{\text {node }}$ stimuli, for a total of 32 stimuli).

\section{Procedure}

All participants completed eight experimental trials, and each experimental trial comprised two stages. In the initial stage one of each of the four stimulus classes was presented in a $2 \times 2$ grid on the computer screen (in much the same manner as Figure 2). The location of each stimulus class within the four quadrants was randomized across trials (unsuccessfully; see Discussion below), and the combination of the four stimulus classes shown on each trial was randomized across participants. The participants were instructed to choose the stimulus that they believed would be the easiest to solve optimally. No limit was placed on the amount of time needed to make a choice. The participants made their choice by clicking on the relevant stimulus using the mouse cursor.

Once the participant had made their choice, the trial moved onto the second stage, in which the four stimuli were replaced by the chosen stimulus, presented in a $17 \times 17 \mathrm{~cm}$ white square located at the center of the screen. The participants were then asked to provide a solution to the given stimulus. Although we were only really interested in the participants' choice frequency data, we felt it was necessary for the participants to generate solutions to the stimuli as well. We reasoned that if the participants were required to solve the problem they chose then they would be more motivated to choose the problem that they felt would be easiest to solve. Similarly, prior to beginning the experiment the participants completed four TSPs for the sake of task familiarization. The practice stimuli were randomly drawn from the medium number of intersections and medium number of hull nodes (i.e., $M_{\text {int }} \& M_{\text {node }}$ ) stimulus class from Vickers et al. (2003) in other words the practice stimuli were independent of the stimuli employed in the main experimental task.

\section{RESULTS}

\section{Choice time}

The participants spent an average of 32.24 seconds (s.d. = 44.61) in making their choices.

\section{Choice probabilities}

Repeated measures analysis of variance indicated a strong and significant main effect of number of nodes on the convex hull $\left(F[1,36]=13.26, p<0.01\right.$, partial $\left.\eta^{2}=0.29\right)$, and a strong and significant main effect of number of intersections $\left(F[1,36]=17.72, p<0.01\right.$, partial $\left.\eta^{2}=0.33\right)$, but no significant interaction $\left(F[1,36]=0.00, p=1.00\right.$, partial $\left.\eta^{2}<0.01\right)$. Importantly, the pattern of effects follows that which could be expected given the empirical data reported in Vickers et al. (2003). Figure 5 shows the Choice Probability data from the current experiment-as can be seen, stimuli with a high number of intersections were chosen more frequently than stimuli with a low number of intersections, and stimuli with a low number of hull nodes were chosen more frequently than stimuli with a high number of hull nodes.

\section{DISCUSSION}

The results of Experiment One follow the expected pattern of results-the participants were most likely to choose to solve stimuli that had previously been shown to be relatively easy, and least likely to choose to solve stimuli that had previously been shown to be relatively difficult. Furthermore, the data suggest that the participants were able to make this discrimination of relative difficulty quite quickly (in around $30 \mathrm{sec}-$ onds on average).

Unfortunately, due to a coding error the assignment of the stimulus classes across the four quadrants was not uniform. Specifically, the least frequently chosen stimulus class $\left(\mathrm{L}_{\mathrm{int}}{ }^{-}\right.$ $\mathrm{H}_{\text {node }}$ ) appeared in the bottom left quadrant with a much 
higher frequency than the other stimulus classes. As a result, although it seems unlikely, we were not able to rule out the possibility that the results of the experiment were due to the participants showing a preference for choosing stimuli based upon their display position. In order to control for this potential confound we repeated the experiment ensuring that the assignment of the stimulus types to the four display locations was uniform. In the following we present the amended replication of the experiment

\section{EXPERIMENT TWO}

Experiment Two replicated Experiment One but controlled for the potential effects of stimulus presentation location upon choice frequency.

\section{Figure 5.}

Empirical choice probability data for the $L_{\text {int }} \& L_{\text {node' }} H_{\text {int }} \& L_{\text {node }}$ $\mathrm{H}_{\text {int }}$ \& $\mathrm{H}_{\text {node' }}$ and $\mathrm{L}_{\text {int }}$ \& $\mathrm{H}_{\text {node }}$ stimulus conditions in Experiment 1 .

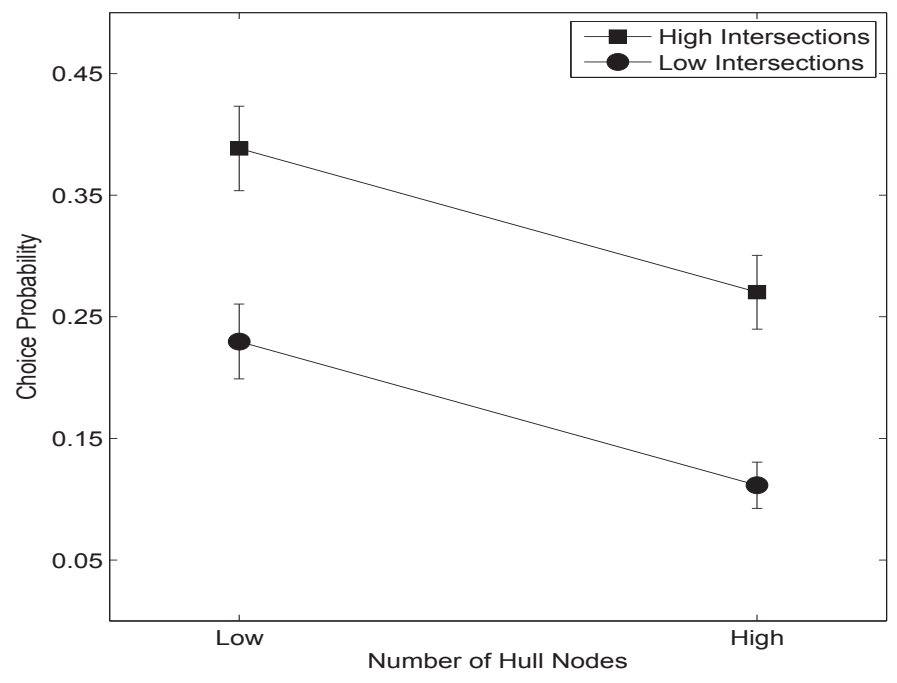

Figure 6.

Empirical choice probability data for the $L_{\text {int }} \& L_{\text {node }} H_{\text {int }} \& L_{\text {node }}$ $\mathrm{H}_{\mathrm{int}} \& \mathrm{H}_{\text {node }}$ and $\mathrm{L}_{\text {int }}$ \& $\mathrm{H}_{\text {node }}$ stimulus conditions in Experiment 2.

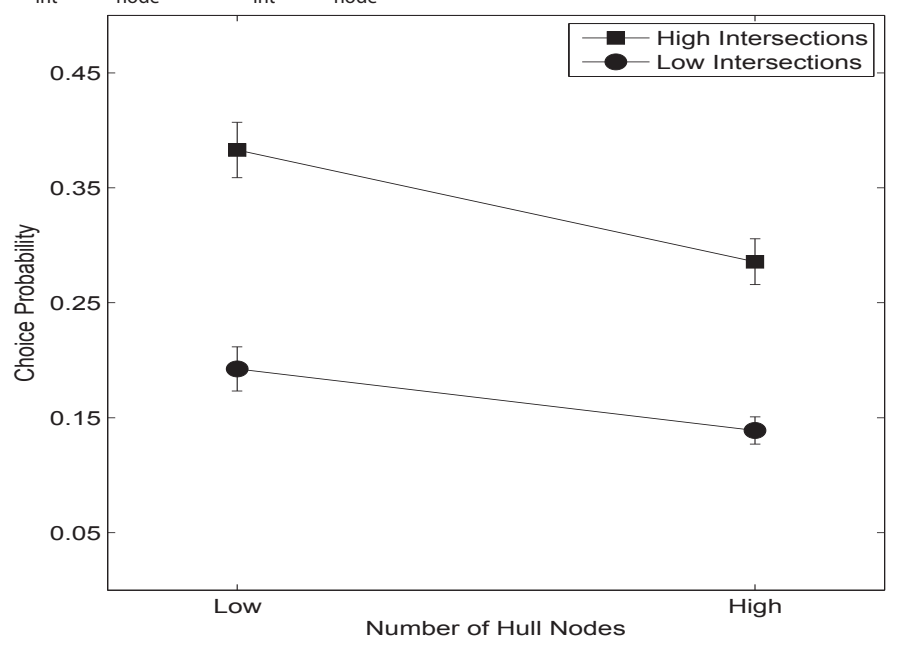

\section{METHOD}

\section{Participants}

The participants $(\mathrm{N}=63)$ were 24 males and 39 females, with a mean age of 26 years. They either performed the experiment for partial course credit or were paid \$15 AUS. All participants provided informed consent and the study was approved by the School of Psychology's human ethics subcommittee.

Stimuli

The stimuli were identical to those employed in Experiment One.

\section{Procedure}

The procedure was identical to that of Experiment One, with the exception that the four stimulus classes were now pseudo-randomly assigned to the four display locations with uniform frequency. Across the 63 participants each of the four stimulus classes was presented in each display quadrant exactly 126 times.

\section{RESULTS}

\section{Choice time}

The participants spent an average of 35.62 seconds (s.d. = 41.51) in making their choices.

\section{Choice probability}

As can be seen in Figure 6, the results of Experiment Two replicate those of Experiment One, with participants choosing the high intersection stimuli more frequently than the low intersection stimuli, and choosing the low hull node stimuli more frequently than the high hull node stimuli. A repeated measures analysis of variance supported this interpretation showing a strong and significant main effect of number of nodes on the convex hull $\left(F[1,62]=13.78, p<0.01\right.$, partial $\eta^{2}$ $=0.18$ ), and a strong and significant main effect of number of potential intersections $\left(F[1,62]=44.35, p<0.01\right.$, partial $\eta^{2}=$ $0.42)$, but no significant interaction $(F[1,62]=1.03, p=0.31$, partial $\eta^{2}=0.02$ ).

\section{DISCUSSION}

The results of Experiment Two closely replicated those of Experiment One. Importantly, given that in Experiment Two the four stimulus classes were uniformly distributed across the four display locations it is safe to rule out the possibility that the choice frequencies were influenced by display location.

\section{ANALYSIS OF STIMULUS PROPERTIES}

The results of the two experiments show a clear pattern of choice probabilities across the four stimulus classes that 
appear to match the relative difficulties of these stimulus classes as reported in Vickers et al. (2003). In the following we present a series of analyses exploring the potential roles of a number of additional stimulus properties that may have contributed to this pattern of results.

As Vickers et al. (2003) note their findings were somewhat unexpected in that the reported relationship between performance quality and number of nodes on the convex hull was actually the opposite of that which had been reported in earlier studies. For example, the results of MacGregor and Ormerod (1996) suggest that higher numbers of convex hull nodes lead to superior task performance. One potential explanation for the discrepancy between these two sets of findings is that there are important differences in the types of stimuli employed in the two experiments. As noted in Lee and Vickers (2000), the stimuli employed in MacGregor and Ormerod (1996) were not randomly generated, had a relatively low node numerosity, and had a high number of nodes that were distributed in a circular fashion on or near the convex hull. In contrast the Vickers et al. stimuli had a much higher numerosity and were randomly distributed. As such, it seems plausible to suggest that the properties affecting problem difficulty in the one set of stimuli might not necessarily affect problem difficulty in the other.

An alternative explanation for this discrepancy is that the intersection/hull node manipulation employed in Vickers et al. (2003) may have inadvertently lead to the manipulation of a third variable that influenced the relative difficulty of the stimuli. One possible reason in support of this hypothesis is that number of intersections and number of convex hull nodes are actually positively correlated (for 10,000 randomly generated 40 node stimuli $\mathrm{r}=0.31, \mathrm{p}<0.001$ ), and this relationship is clearly discernable in Figure 1 of Vickers et al. (2003). Given this relationship we might expect that (all other things being equal) number of intersections and number of convex hull nodes might both be positively related to TSP performance quality.

In order to test this we re-analysed the stimuli employed in these experiments to determine if there were any additional systematic differences between the stimulus classes that might explain the results of Vickers et al. (2003) and the current experiment. We focused upon five properties of TSP stimuli or their associated optimal solutions: degree of clustering/regularity, convexity of optimal solution, circularity of optimal solution, number of indentations in the optimal solution, and path complexity of optimal solution.

Only the first of these properties has been shown to directly affect empirical TSP performance: in Dry, Preiss, and Wagemanns (2012) it was demonstrated that highly clustered stimuli are easier to solve than randomly distributed stimuli, which are in turn easier to solve than regularly distributed stimuli. The degree of relative clustering/regularity is measured as $\mathrm{R}$ which is equal to 1 for randomly distributed stimuli, approaches 0 as the degree of clustering increases, and approaches 2.149 as stimuli become increasingly regular in their node distribution.

Convexity of optimal solution, circularity of optimal solution, and number of indentations in the optimal solution can be thought of as measures of the degree to which an optimal solution deviates from the stimulus convex hull. Degree of convexity is measured as a ratio of the area enclosed by the optimal solution to the area enclosed by the convex hull. Circularity is measured as $4 \pi \mathrm{A} / \mathrm{p}^{2}$, where $\mathrm{A}$ is the area enclosed by the solution and $\mathrm{p}$ is the length of the solution. For both convexity and circularity high values indicate that the solution tends to follow the convex hull, and low values indicate deviation from the hull. The number of indentations is measured as the number of times that adjacent nodes on the convex hull are not directly connected in the optimal solution. In this case high values indicate high deviation from the convex hull and low values indicate low deviation. As has been indicated, none of these properties have been directly linked to the quality of human performance on TSPs, however MacGregor (2012) demonstrated that participants show a bias towards generating fewer indentations in their solutions than are actually contained in the optimal solution, and Vickers, Lee, Dry, Hughes, and MacMahon (2006) demonstrated that both circularity and convexity are good predictors of the aesthetic appeal of TSP solutions. In any case, if participants are employing the convex hull as a guide for generating TSP solutions then it could be assumed that TSP stimuli with optimal solutions that deviate highly from the hull would be harder to solve than stimuli with optimal solutions that follow the hull more closely.

Finally, path complexity is a measure of the neighbour-relations of the inter-node connections in an optimal solution. Each edge in a solution is assigned a value (1 through $\mathrm{k}$ ) according to whether the internode connection represented by the edge joins $1^{\text {st }}$ through $\mathrm{k}^{\text {th }}$ neighbouring nodes, and these values are then averaged. Stimuli with a low path complexity have optimal solutions that are comprised of connections between nodes that are nearest (or low order) neighbours. Vickers et al. (2006) demonstrated that path complexity is a significant predictor of the aesthetic appeal of TSP solutions, and Vickers, Mayo, Heitmann, Lee, and Hughes (2004) demonstrated that empirical solutions with low path complexity tended to be closer to the optimal solution than empirical solutions with high path complexity. In light of this it seems reasonable to assume that TSP stimuli that have optimal solutions with low path complexity would be easier to solve than stimuli with high complexity optimal solutions.

Table 1 shows the values of the five stimulus properties for each of the stimulus classes. Univariate analysis of variance indicated no significant differences between the stimuli 
Table 1.

Comparison of stimulus properties for the four stimulus classes.

\begin{tabular}{|l|c|c|c|c|}
\hline & $\mathrm{H}^{\text {int }} \& \mathrm{~L}^{\text {node }}$ & $\mathrm{H}^{\text {int }} \& \mathrm{H}^{\text {node }}$ & $\mathrm{L}^{\text {int }} \& \mathrm{~L}^{\text {node }}$ & $\mathrm{L}^{\text {int }} \& \mathrm{H}^{\text {node }}$ \\
\hline R-value (clustering/regularity) & 0.96 & 1.02 & 0.99 & 0.99 \\
\hline Convexity & 0.62 & 0.62 & 0.52 & 0.59 \\
\hline Circularity & 1292 & 766 & 660 & 891 \\
\hline Number of Indentations & 5.75 & 7.50 & 5.50 & 6.62 \\
\hline Path Complexity & 4.78 & 4.49 & 4.79 & 4.66 \\
\hline
\end{tabular}

in terms of R-value, circularity, or path complexity. However, in regards to number of indentations we found that there was a significant main effect of hull nodes $(F[1,28]=13.86, p$ $<0.001)$. Specifically, the stimuli with a low number of nodes on the convex hull had optimal solutions with significantly fewer indentations compared to the stimuli with a high number of hull nodes. We also found a significant interaction effect for convexity $(\mathrm{F}[1,28]=4.81, \mathrm{p}<0.036)$; specifically, the optimal solutions for $\mathrm{L}_{\text {int }} \& \mathrm{~L}_{\text {node }}$ stimuli had significantly lower convexity values than those of the $\mathrm{H}_{\text {int }} \& \mathrm{~L}_{\text {node }}$, $\mathrm{L}_{\text {int }}$ \& $\mathrm{H}_{\text {node }}$, and $\mathrm{H}_{\text {int }} \& \mathrm{H}_{\text {node }}$ stimuli $(\mathrm{t}[14]=4.33,2.64$, and 3.83, respectively, all $\mathrm{p}<0.05$ ).

It is difficult to see how results concerning optimal solution convexity could explain the relationship between number of hull nodes and solution difficulty as only one of the stimulus classes differed significantly in this regard. Furthermore, in theory stimuli with low convexity values should be harder to solve as their optimal solutions deviate more from the hull than stimuli with high convexity values. However, the data from Vickers et al. (2003) indicate that the $\mathrm{L}_{\text {int }} \& \mathrm{~L}_{\text {node }}$ stimuli were not the hardest to solve relative to the other stimulus classes, and the data from the current experiments indicates that the $\mathrm{L}_{\text {int }} \& \mathrm{~L}_{\text {node }}$ stimuli were not the least frequently chosen stimuli in regards to perceived solution difficulty.

In contrast, the finding concerning number of indentations has more potential as an explanation as in this case the pattern of significant differences matches the observed pattern of solution difficulty and choice frequency more closely. Further, it is possible to interpret the pattern of differences in a theoretically plausible manner. As indicated, the results of MacGregor (2012) suggest that human solvers have a tendency to produce solutions that have few indentations, and that their solutions tend to have fewer indentations than the associated optimal solution. Following this it seems reasonable to assume that stimuli with optimal solutions that contain a high numbers of indentations would be relatively more difficult as the inherent bias of human solvers towards producing low indentation pathways would result in sub-optimal solutions. Hence, the observed pattern of results suggesting that the high hull node stimuli were more difficult to solve than the low hull node stimuli in Vickers et al. (2003), and were chosen less frequently than the low hull node stimuli in the current experiments.

Additionally, the properties of the stimuli themselves indicate that logically the high hull node stimuli should have (on average) optimal solutions with a high number of indentations compared to the low node stimuli. Specifically, the $\mathrm{H}_{\text {int }} \& \mathrm{~L}_{\text {node }}$ and $\mathrm{L}_{\text {int }} \& \mathrm{~L}_{\text {node }}$ stimuli had 8 nodes on the hull and 32 nodes within the hull-in this case the minimum number of potential indentations is 1 , and the maximum number of indentations is 8 . In contrast, the $\mathrm{L}_{\text {int }} \& \mathrm{H}_{\text {node }}$, and $\mathrm{H}_{\text {int }}$ \& $\mathrm{H}_{\text {node }}$ had 12 nodes on the hull and 28 within-in this case the minimum number of potential indentations is still 1 , but the maximum is 12 .

We can draw two conclusions from these analyses. First, they indicate that the stimuli employed in Vickers et al. (2003) and the current study did not only differ in regards to the number of nodes on the convex hull and number of indentations. As Table 1 indicates, the stimuli differed along a range of different properties, and in some cases these differences were statistically significant. It is important to note that the properties explored in our analyses are by no means exhaustive, and it is possible that the stimuli also differed in terms of other properties which may have influenced their relative difficulty.

Second, the finding regarding number of indentations provides some insight into the unexpected results of Vickers et al. (2003) regarding a negative relationship between hull node numerosity and solution difficulty, and the similar findings in the current study regarding a negative relationship between hull node numerosity and choice frequency. While the results of MacGregor (2012) indicate a general bias towards generating TSP solutions with a low number of indentations it would be highly interesting to test whether there is a difference between the solution difficulties of TSP stimuli that have optimal solutions with high, medium or low numbers of indentations.

\section{GENERAL DISCUSSION}

In this paper we have presented two experiments demonstrating that human observers are able to make fast and 
efficient discriminations of the relative difficulty of TSP stimuli. Further, we have provided further insight into the underlying properties that appear to govern the solution difficulty of TSP stimuli. In the following we discuss the wider implications of these findings.

As indicated, the results of Experiments One and Two demonstrate that when participants are presented with sets of four TSP stimuli that have been previously shown to vary in terms of their solution difficulty they are able to systematically differentiate between them. Specifically, the mean patterns of choice frequency in the current experiments closely matched the mean patterns of solution difficulty found in Vickers et al. (2003).

Anecdotal evidence from Dry et al. (2012) suggests that participants are aware of the varying difficulty of different TSP stimuli. In their study a number of participants made unprompted remarks once they had completed the experiment indicating that they had found the highly clustered stimuli easier to solve than the other stimuli, and this matched the actual observed pattern of solution difficulty. The results of the current experiments are remarkable for two reasons. First, unlike the clustered-random-regular stimuli employed in Dry et al. (2012) which differed in an obvious qualitative manner, Figure 2 demonstrates that there were no easily discernable differences between the stimuli employed in our experiments. Second, in Dry et al. the participants made comments regarding the varying difficulty of the stimuli after they had provided solutions to them. In our study the participants were able to make meaningful differentiations between the stimuli before they had physically tackled the problems.

It is unclear how they were able to actually perform this discrimination. One possibility is that they generated solutions or partial solutions for all four of the stimuli, stored these in working memory and compared them to each other in regards to perceived optimality or ease of solution generation, or both. If this were the case then it would appear that the processing ability of human solvers in regards to TSPs is more sophisticated and/or powerful than previously assumed. It should also be noted that the participants were able to make these discriminations in around 30 seconds, which is approximately one third of the length of time required to physically produce solutions to problems of equal numerosity as reported in Dry et al. (2006).

We believe that the speed and efficiency of discrimination reported in our experiments provides further evidence in favour of the suggestion that some form of automatic perceptual processing may be involved in human TSP performance (e.g., MacGregor \& Ormerod, 1996; van Rooij, Schactman, Kadlec, \& Stege, 2006; Vickers et al., 2003; Vickers et al., 2006). Specifically, it has been suggested that this process is an example of the law of Prägnanz (or minimum principle) which proposed that the visual system is inherently biased towards perceiving structure that is minimal (Köhler, 1929). In other words, it would seem that humans are able to generate optimal or close-to-optimal solutions to TSPs because these solutions represent the type of minimal structures that the visual system inherently and automatically perceives.

Evidence supporting this can be found in a number of sources, for example in Vickers et al. (2001) they asked two groups of participants to solve TSPs using very different methods: one group was asked to find the optimal solution to the TSPs (i.e., find the shortest pathway), while the other group was instructed to create a pathway that "looked natural, attractive, or aesthetically pleasing." A comparison of the resulting pathways indicated that there was a high degree of overlap between the tours produced by the two groups. Further, in Pomerantz (1981) and Dry, Navarro, Preiss, and Lee (2009) participants were presented with dot arrays and asked to join up the dots to show the structure that they perceived. In each case the empirical structures tended towards the simplest possible configuration of the point set. In other words, the results of these studies suggest even when participants are not specifically told to find the minimal structure in the stimuli they behave as if they had been.

In regards to the current experiments, it is interesting to note that both of the stimulus properties of interest can be thought of as representing a form of geometric minimality. For example, MacGregor and Ormerod (1996) suggest that the convex hull may represent "a two-dimensional illustration of the law of Prägnanz in the same way that soap bubbles represent it in three". Similarly, Vickers et al. (2003) relates intersecting edges (or more rightly, non-intersecting edges) to minimal structures such as the inter-node neighbor-relations found in Voronoi tessellations (e.g., Ahuja, 1982; Dry et al., 2009). In other words, if the participants were implicitly aware of these sorts of minimal structures that are present in the stimuli, then this may have helped to guide them in their discriminations. Obviously this suggestion is highly conjectural and requires further investigation, but we believe that at the very least it represents a plausible potential explanation for the findings of the current study, and those of previous studies.

Finally, it is important to note that the results of the stimulus analyses we performed suggest that the number of convex hull nodes and number of intersecting edges may not have been the only properties influencing the relative solution difficulty of the stimuli. Our analyses indicated that there were indeed statistically significant differences between the geometric properties of the stimulus classes, and in the case of one of these properties (number of indentations) it was possible to posit a potential explanation for the discrepancy between the results of Vickers et al. (2003) and those of earlier studies such as MacGregor and Ormerod (1996). In light of these findings we are currently exploring the interrelationships between these different stimulus properties and 
the influence that they have upon TSP solution difficulty. It is hoped that these investigations will provide further insight into the results of the current study, and ultimately lead to a better understanding of the processes underlying human performance on this computationally difficult task.

\section{NOTES}

1. We thank Tom Ormerod for pointing out this alternative theoretical hypothesis.

2. We discuss the disparity between the results of Vickers et al. (2003) regarding solution difficulty and number of hull nodes, and the results of other studies (e.g., MacGregor \& Ormerod, 1996) in the Analysis of Stimulus Properties section of this paper

\section{REFERENCES}

Ahuja, N. (1982). Dot pattern processing using Voronoi neighborhoods. IEEE Transactions on Pattern Analysis and Machine Intelligence, 4(3), 336-343. http://dx.doi.org /10.1109/TPAMI.1982.4767255

Best, B. J. (2005). A model of fast human performance on a computationally hard problem. In Proceedings of th 27th Annual Conference of the Cognitive Science Society (pp. 256-251). Austin, TX: Cognitive Science Society.

Dry, M. J., Lee, M. D., Vickers, D., \& Hughes, P. (2006). Human performance on visually presented traveling salesperson problems with varying numbers of nodes. The Journal of Problem Solving, 1(1), 20-32. http://dx.doi.org /10.7771/1932-6246.1004

Dry, M. J., Navarro, D. J., Preiss, K., \& Lee, M. D. (2009). The perceptual organization of point constellations. In N. A. Taatgen \& H. v. Rijn (Eds.), Proceedings of the 31st Annual Conference of the Cognitive Science Society (pp. 11511156). Austin, TX: Cognitive Science Society.

Dry, M. J., Preiss, K., \& Wagemans, J. (2012). Clustering, randomness and regularity: Spatial distributions and human performance on the traveling salesperson problem and minimum spanning tree problem. Journal of Problem Solving, 4(1), 1-17. http://dx.doi.org/10.7771/1932-6246.1117

Garfinkel, R. S. (1985). Motivation and modeling. In E. L. Lawler, J. K. Lenstra, A. H. G. Rinnooy \& D. B. Shmoys (Eds.), The traveling salesman problem: A guided tour of combinatorial optimization. London, UK: John Wiley \& Sons.

Graham, S. M., Joshi, A., \& Pizlo, Z. (2000). The traveling salesman problem: A hierarchical model. Memory \& Cognition, 28(7), 1191-1204. http://dx.doi.org/10.3758 /BF03211820

Haxhimusa, Y., Carpenter, E., Catrambone, J., Foldes, D., Stefanov, E., Arns, L., \& Pizlo, Z. (2011). 2D and 3D traveling salesman problem. The Journal of Problem Solving, 3(2), 167-193. http://dx.doi.org/10.7771/1932-6246.1096

Haxhimusa, Y., Kropatsch, W. G., Pizlo, Z., \& Ion, A. (2009). Approximate graph pyramid solution of the E-TSP. Image and Vision Computing, 27(7), 887-896. http://dx.doi.org /10.1016/j.imavis.2008.06.016

Hill, A. V. (1982). An experimental comparison of human schedulers and heuristic algorithms for the traveling salesman problem. JournalofOperations Management, 2(4),215223. http://dx.doi.org/10.1016/0272-6963(82)90010-9

Köhler, W. (1929). Gestalt psychology. New York, NY: Liveright. Lee, M. D., \& Vickers, D. (2000). The importance of the convex hull for human performance on the traveling salesman problem: A comment on MacGregor and Ormerod (1996). Perception \& Psychophysics, 62(1), 226-228. http:// dx.doi.org/10.3758/BF03212074

MacGregor, J. N. (2012). Indentations and starting points in traveling sales tour problems: Implications for theory. Journal of Problem Solving, 5(1), 2-17. http://dx.doi.org /10.7771/1932-6246.1140

MacGregor, J. N., \& Ormerod, T. C. (1996). Human performance on the traveling salesman problem. Perception \& Psychophysics, 58(4), 527-539. http://dx.doi.org/10.3758 /BF03213088

MacGregor, J. N., Ormerod, T. C., \& Chronicle, E. P. (1999). Spatial and contextual factors in human performance on the traveling salesperson problem. Perception, 28(11), 1417-1427. http://dx.doi.org/10.1068/p2863

MacGregor, J. N., Ormerod, T. C., \& Chronicle, E. P. (2000). A model of human performance on the traveling salesperson problem. Memory \& Cognition, 28(7), 1183-1190. http://dx.doi.org/10.3758/BF03211819

Pizlo, Z., Stefanov, E., Saalweachter, J., Li, Z., Haxhimusa, Y., \& Kropatsch, W. G. (2006). Traveling salesperson problem: A foveating pyramid model. The Journal of Problem Solving, 1(1), 83-101. http://dx.doi.org/10.7771/1932-6246.1009

Pomerantz, J. R. (1981). Perceptual organization in information processing. In M. Kubovy \& J. R. Pomerantz (Eds.), Perceptual organization (pp. 141-180). Mahweh, NJ: Lawrence Erlbaum.

Tak, S., Plaiser, M., \& van Rooij, I. (2008). Some tours are more equal than others: The convex hull model revisited with lessons for testing models of the traveling salesperson problem. The Journal of Problem Solving, 2(1), 4-28. http://dx.doi.org/10.7771/1932-6246.1028

van Rooij, I., Schactman, A., Kadlec, H., \& Stege, U. (2006). Perceptual or analytical processing? Evidence from children's and adult's performance on the Euclidean traveling salesperson problem. The Journal of Problem Solving, 1(1), 44-73. http://dx.doi.org/10.7771/1932-6246.1006

van Rooij, I., Stege, U., \& Schactman, A. (2003). Convex hull and tour crossings in the Euclidean traveling salesperson 
problem: Implications for human performance studies. Memory \& Cognition, 31(2), 215-220. http://dx.doi.org /10.3758/BF03194380

Vickers, D., Butavicius, M., Lee, M., \& Medvedev, A. (2001). Human performance on visually presented traveling salesman problems. Psychological Research, 65(1), 34-45. http://dx.doi.org/10.1007/s004260000031

Vickers, D., Lee, M. D., Dry, M. J., \& Hughes, P. (2003). The roles of the convex hull and the number of potential intersections in performance on visually presented traveling salesperson problems. Memory \& Cognition, 31(7), 10941104. http://dx.doi.org/10.3758/BF03196130

Vickers, D., Lee, M. D., Dry, M. J., Hughes, P., \& McMahon, J. A. (2006). The aesthetic appeal of minimal structures:
Judging the attractiveness of solutions to traveling salesperson problems. Perception \& Psychophysics, 68(1), 3242. http://dx.doi.org/10.3758/BF03193653

Vickers, D., Mayo, T., Heitmann, M., Lee, M., \& Hughes, P. (2004). Intelligence and individual differences in performance on three types of visually presented optimisation problems. Personality and Individual Differences, 36(5), 1059-1071. http://dx.doi.org/10.1016 /S0191-8869(03)00200-9

Walwyn, A. L., \& Navarro, D. J. (2010). Minimal paths in the city block: Human performance on Euclidean and non-Euclidean traveling salesperson problems. The Journal of Problem Solving, 3(1), 93-105. http://dx.doi.org /10.7771/1932-6246.1080 